\title{
Modern contraceptive use among women in need of family planning in India: an analysis of the inequalities related to the mix of methods used
}

Fernanda Ewerling ${ }^{1,5^{*}}(0)$, Lotus McDougal ${ }^{2}$, Anita Raj ${ }^{2}$, Leonardo Z. Ferreira ${ }^{1,3}$, Cauane Blumenberg ${ }^{1}$, Divya Parmar ${ }^{4}$ and Aluisio J. D. Barros ${ }^{1,3}$

\begin{abstract}
Objective: To evaluate the type of contraceptives used by women in need of family planning in India and the inequalities associated with that use according to women's age, education, wealth, subnational region of residence and empowerment level.

Methods: Using data from the Indian National Family and Health Survey-4 (2015-2016), we evaluated the proportion of partnered women aged 15-49 years with demand for family planning satisfied (DFPS) with modern contraceptive methods. We also explored the share of each type of contraception [short- (e.g., condom, pill) and long-acting (i.e., IUD) reversible contraceptives and permanent methods] and related inequalities.

Results: The majority $(71.8 \%$; $95 \% \mathrm{Cl} 71.4-72.2)$ of women in need of contraception were using a modern method, most (76.1\%) in the form of female sterilization. Condom and contraceptive pill were the second and third most frequently used methods (11.8\% and $8.5 \%$, respectively); only 3.2\% reported IUD. There was a nearly linear exchange from short-acting to permanent contraceptive methods as women aged. Women in the poorest wealth quintile had DFPS with modern methods at least 10 percentage points lower than other women. We observed wide geographic variation in DFPS with modern contraceptives, ranging from $23.6 \%(95 \% \mathrm{Cl} 22.1-25.2)$ in Manipur to $93.6 \%(95 \% \mathrm{Cl}$ 92.8-94.3) in Andhra Pradesh. Women with more accepting attitudes towards domestic violence and lower levels of social independence had higher DFPS with modern methods but also had higher reliance on permanent methods. Among sterilized women, 43.2\% (95\% Cl 42.7-43.7) were sterilized before age 25, 61.5\% (95\% Cl 61.0-62.1) received monetary compensation for sterilization, and $20.8 \%(95 \% \mathrm{Cl} 20.3-21.3)$ were not informed that sterilization prevented future pregnancies.
\end{abstract}

Conclusion: Indian family planning policy should prioritize women-centered care, making reversible contraceptive methods widely available and promoted.

*Correspondence: fewerling@equidade.org

${ }^{5}$ Marechal Deodoro 1160, 3rd floor, Pelotas, RS, Brazil

Full list of author information is available at the end of the article

(c) The Author(s) 2021. Open Access This article is licensed under a Creative Commons Attribution 4.0 International License, which permits use, sharing, adaptation, distribution and reproduction in any medium or format, as long as you give appropriate credit to the original author(s) and the source, provide a link to the Creative Commons licence, and indicate if changes were made. The images or other third party material in this article are included in the article's Creative Commons licence, unless indicated otherwise in a credit line to the material. If material is not included in the article's Creative Commons licence and your intended use is not permitted by statutory regulation or exceeds the permitted use, you will need to obtain permission directly from the copyright holder. To view a copy of this licence, visit http://creativecommons.org/licenses/by/4.0/. The Creative Commons Public Domain Dedication waiver (http://creativeco mmons.org/publicdomain/zero/1.0/) applies to the data made available in this article, unless otherwise stated in a credit line to the data. 


\begin{abstract}
Plain Language Summary
Ensuring universal access to sexual and reproductive health and reproductive rights for all women is one of the Sustainable Development Goals, promoted by the United Nations and adopted by 193 countries, including India. To address women's need for contraception, the provision of a wide range of safe, effective and affordable contraceptive methods is essential. In this study, we evaluated the type of contraceptives used by women in need of contraception in India and the inequalities in contraceptive use among different subgroups of women. The majority (71.8\%) of women in need of contraception were using a modern method, most (76.1\%) in the form of female sterilization. Condom and contraceptive pill were the second and third most frequently used methods $(11.8 \%$ and $8.5 \%$, respectively); only 3.2\% reported IUD. We observed an exchange from short-acting to permanent contraceptive methods as women aged. The poorest women presented demand for family planning satisfied (DFPS) with modern methods at least 10 percentage points lower than other women. There was wide geographic variation in DFPS with modern contraceptives, ranging from 23.6\% in Manipur to 93.6\% in Andhra Pradesh. Women with more accepting attitudes towards domestic violence and lower levels of social independence had higher DFPS with modern methods but also had higher reliance on permanent methods. Among sterilized women, $43.2 \%$ were sterilized before age $25,61.5 \%$ received monetary compensation for sterilization, and $20.8 \%$ were not informed that sterilization prevented future pregnancies. Indian family planning policy should prioritize women-centered care, making reversible contraceptive methods widely available and promoted.
\end{abstract}

Keywords: Family planning, Contraceptive use, Health inequalities

\section{Introduction}

Ensuring universal access to sexual and reproductive health and reproductive rights for all women is Target 5.6 of the Sustainable Development Goals, promoted by the United Nations and adopted by 193 countries. To address women's need for family planning, the provision of a wide range of safe, effective and affordable contraceptive methods is essential [1]. The mix of methods offered must cater to women's needs and preferences. It is also important to note that every contraceptive method has advantages and disadvantages [2]. Thus, it is essential that women are fully informed about them so they can make an informed decision on which method is more appropriate for their specific situation. A nationally representative study found that India's demand for family planning satisfied (DFPS) with modern contraceptive methods was $70 \%$ in 2005 , with heavy reliance on female sterilization rather than reversible contraceptive methods [3]. These findings already suggested the need for greater focus on improving access to reversible methods, especially for women who wish to delay or space pregnancies but are not ready to commit to ending their fertility.

Family planning policies in India have historically been aimed at controlling population growth rather than advancing women's reproductive rights and choices [4, 5]. This led to an explicit promotion of sterilization, targeted nearly exclusively towards women. Government policy has since changed, as laid out in the 2014 Family Planning 2020 action plan [6], which still promotes sterilization with monetary compensation (both for individuals undergoing the procedure and for the health providers) but also includes reversible modern contraception methods. At this time, three new contraceptive methods were introduced in the National Family Planning program-injectable contraceptive, a non-hormonal weekly pill and progesterone-only pills for lactating mothers-all provided free-of cost. Intrauterine device (IUD) remains low despite being covered under public health services for decades and condom use only increased subsequent to HIV prevention efforts in the country. However, despite these changes in the policy environment, the use of reversible contraceptive methods, as well as male sterilization, is still low in India, and female sterilization continues to be the dominant method, accounting for two-thirds of the total contraceptive use [1, 7].

In this paper, we evaluated the coverage of DFPS with modern methods in India and the share of each type of contraception (long-acting reversible, short-acting reversible and permanent methods) being used. Additionally, we assessed inequalities in these indicators according to women's age, education, wealth, subnational region of residence and empowerment level.

\section{Methods}

We used data from the National Family and Health Survey (NFHS-4) conducted in India in 2015-2016. This survey was implemented using a multistage sampling strategy. In the first stage, 28,586 primary sampling units (PSU) were selected with a probability of selection proportional to the PSU size. The selected PSUs were mapped, and their households were listed. In the second stage, 22 households from each PSU were systematically selected, totaling 628,892 households included in the sample. All women aged 15-49 years who slept 
in the selected households the night before the interview were invited to participate in the survey. In total, 699,686 women were interviewed on topics including family planning. An extended version of the questionnaire was applied to a subsample of 112,351 women, including, among others, questions related to women's empowerment and domestic violence. This subsample is representative at state-level, while the larger sample is representative at district level [8].

Our main outcome of interest is DFPS with modern methods among women aged 15-49 years who are currently married or in union (hereafter referred to as partnered women). This indicator is defined as the proportion of women using a modern contraceptive method among those in need of contraception (women who are fecund and do not want to become pregnant within the next 2 years, or who are unsure about whether or when they want to become pregnant) [9]. Women are considered infecund if they (1) are married for five years or more, did not use contraception and had not gotten pregnant in that period; (2) reported that they cannot get pregnant; (3) reported menopause, hysterectomy or never menstruated; or (4) had last period more than 6 months ago and are not postpartum amenorrheic. According to the indicator definition, pregnant women with a mistimed or unwanted pregnancy are also considered in need of family planning [9]. We used the definition of modern contraception proposed by Hubacher and Trussel [10], that classifies modern contraceptive methods as technological products or medical procedures that affect natural reproduction. According to this definition, the following contraceptive methods were considered as modern: contraceptive pills, condoms (male and female), IUD, sterilization (male and female), injectables, diaphragms, spermicidal agents (foam/jelly), and emergency contraception. The modern contraceptive methods analyzed were also classified as: (1) short-acting reversible contraception (SARC), including contraceptive pills, injectables, condoms, diaphragms, spermicidal agents and emergency contraception; (2) long-acting reversible contraception (LARC), including solely IUD; and (3) permanent methods, comprised of male and female sterilization. Hormone implants (LARC) and patches are also considered modern methods but were recorded in the DHS questionnaire as "other modern methods". However, none of the women interviewed reported this category.

We evaluated the proportion of partnered women who have DFPS with modern methods and the share of each type of contraceptive among the users. To better understand the patterns among subgroups, we also stratified the analyses by women's age, education (categorized as none; primary; secondary or higher), household wealth quintiles, based on the asset index included in the survey dataset (Q1 being the poorest and Q5 the richest quintile), subnational region and women's empowerment as measured by the SWPER global index [11]. The SWPER global index is an individual-level indicator based on 14 questions that allows the assessment of three empowerment domains: (1) attitude to domestic violence, which comprises questions related to the women's opinion on whether beating the wife is justified in five situations (if the woman goes out without telling the husband; neglects the children; argues with her husband; refuse to have sex; and if she burns the food); (2) social independence, that includes the women's access to information, educational attainment, age at first marriage and first child, and the difference in age and education between the woman and her husband; and (3) decision making, which is comprised of three questions on who makes decisions in the household in regard to the respondent's health care, major expenses and visits to family and relatives. The SWPER is a cross-culturally tested tool that allows the measurement of women's empowerment at individual-level and among subgroups of women. The resulting scores are categorized as low, medium, and high empowerment level using the cutoffs provided with the SWPER global methodology. Full details on the construction of the index and its validity are presented elsewhere [11].

Given the extremely high DFPS with permanent methods in the country, we further explored the available descriptive information about the women that were sterilized, including: age and parity at sterilization, whether they were told that sterilization would mean no more children, whether they received monetary compensation to undergo the procedure (and the average compensation received) according to wealth quintiles, and whether they regret the sterilization according to their parity at sterilization. These analyses were also restricted to currently partnered women, but the results including all women, regardless of their marital status, were virtually the same (results not shown).

All estimates were calculated taking the survey sample design (including clusters, strata, and sample weights) into account. All analyses were conducted using the statistical software Stata 15 (StataCorp. 2017. Stata Statistical Software: Release 15. College Station, TX: StataCorp LLC). NFHS-4 is a publicly available source of data, so ethical clearance was not required for this study. The NFHS-4 study protocol, including all the survey questionnaires, was approved by the International Institute for Population Sciences 
Institutional Review Board and the ICF Institutional Review Board.

\section{Results}

Among the 339,540 partnered women aged 15-49 years in need of contraception, $71.8 \%$ (95\% CI 71.4-72.2) were using a modern contraceptive method. Table 1 presents

Table 1 Type of contraception being used among partnered women aged 15-49 years currently using contraception in India, 2015-2016 $(\mathrm{N}=243,814)$

\begin{tabular}{lrc}
\hline & $\%$ & $\mathbf{9 5 \%} \mathbf{~ C l}$ \\
\hline Short-acting reversible contraception & 20.7 & $20.3-21.2$ \\
Pill & 8.5 & $8.2-8.9$ \\
Injectables & 0.4 & $0.3-0.4$ \\
Condoms & 11.8 & $11.5-12.1$ \\
Long-acting reversible contraception & 3.2 & $3.1-3.3$ \\
IUD & 3.2 & $3.1-3.3$ \\
Permanent contraception & 76.1 & $75.5-76.6$ \\
Female sterilization & 75.5 & $75.0-76.0$ \\
Male sterilization & 0.6 & $0.5-0.6$ \\
\hline
\end{tabular}

Other short-acting reversible contraceptive methods, such as diaphragm, spermicidal agents (foam/jelly), and emergency contraception were reported by only 9 women $(<0.01 \%)$, thus they were not included in the table the share of contraceptive methods used by women with DFPS with a modern method, showing that most women using contraceptives in India rely on permanent contraception (76.1\%, 95\% CI 75.5-76.6), while 20.7\% (95\% CI 20.3-21.2) are using SARC, and 3.2\% (95\% CI 3.1-3.3) LARC methods. Analyzing the specific method being used, results show that $75.5 \%$ (95\% CI 75.0-76.0) of the women using a modern method are sterilized, while only $0.6 \%$ (95\% CI 0.5-0.6) had male partners who were subjected to a vasectomy. Condom and contraceptive pill were the most used methods after sterilization, being respectively used by $11.8 \%$ (95\% CI $11.5-12.1$ ) and $8.5 \%$ (95\% CI 8.2-8.9) of the women and thus comprising almost all of SARC use. Only 3.2\% (95\% CI 3.1-3.3) relied on LARC (i.e., IUD).

Large disparities in the proportion of partnered women with DFPS with modern methods were observed according to the women's age, with higher coverage among older women (Fig. 1). Women aged 40 years or older presented over three times the DFPS with modern methods when compared to women aged 15 to 19 years of age. Among women using modern methods, there is nearly a linear exchange of short-acting to permanent contraceptive methods as age increases. In the early twenties, around $20 \%$ of the users rely on permanent methods; this share

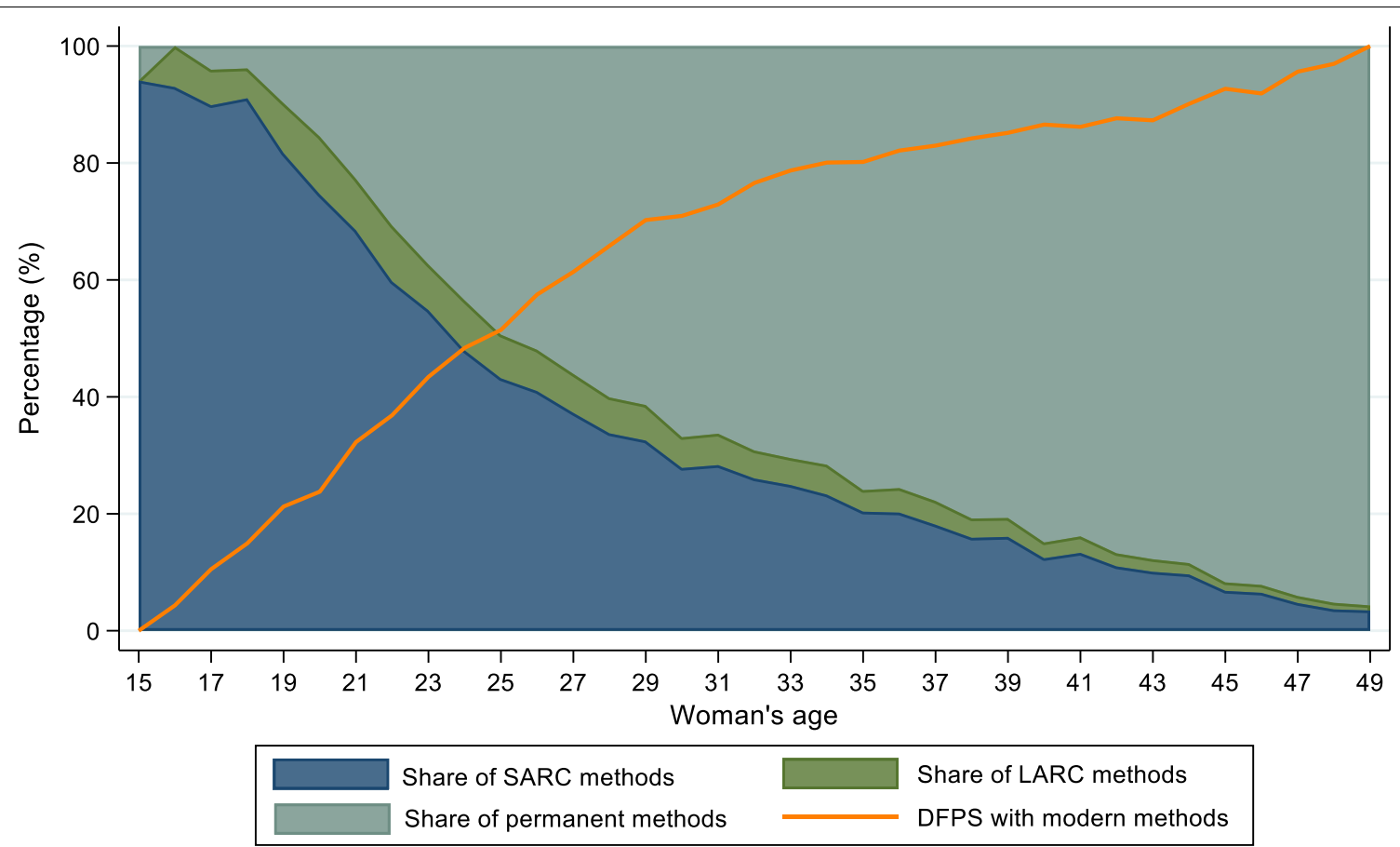

Fig. 1 Demand for family planning satisfied (DFPS) with modern methods and the share of each type of contraceptive method among partnered women aged 15-49 currently using contraception of modern contraception and according to women's age. India, 2015-2016 (N=339,540). Note Short-acting reversible contraceptive (SARC) methods include condoms (male and female), injectables, diaphragms, jelly or foam and emergency contraception; Long-acting reversible contraceptive (LARC) methods include intrauterine devices (IUD); and Permanent methods include male and female sterilization 
quickly rises and reaches $80 \%$ in the forties. The share of LARC is relatively constant at around 5\% in women of 15 to 35 years old, slowly decreasing until the end of reproductive age.

Across household wealth quintiles, women in the poorest quintile lagged behind the rest, with approximately $10 \%$ points lower DFPS, as observed in Fig. 2. Within the richest quintile, there was also a larger share of SARC and LARC methods and a lower reliance on permanent contraception. Figure 3 shows that more educated women had around 10 percentage points lower coverage of DFPS with modern methods when compared to less educated women. However, the share of SARC and LARC methods was much higher among more educated women, while less-educated women more frequently use permanent methods, revealing once again the extreme reliance on sterilization for satisfying family planning needs.

Strong geographic variation can be clearly observed in DFPS with modern methods (Fig. 4A), ranging from 23.6\% (95\% CI 22.1-25.2) in Manipur to 93.6\% (95\% CI 92.8-94.3) in Andhra Pradesh. Southern states, except for Lakshadweep archipelago, present higher coverage when compared to northeastern states and also the highest shares of permanent methods. Generally, reliance on permanent contraception was remarkably high, accounting for over $70 \%$ of modern contraception in 17 out of the 36 Indian states/union territories (Fig. 4D). The share of short-acting contraceptive use only surpasses $50 \%$ in Assam, Chandigarh, Meghalaya and Tripura (Fig. 4B). Long-lasting share fails to reach $5 \%$ in 22 states, and only exceeds 15\% in Manipur and Nagaland (Fig. 4C). Estimates for all states and their confidence intervals are presented in the Additional file 1: Table S1.

According to the empowerment level (using the subsample of women), Fig. 5 shows that women who were more empowered in terms of attitude to violence and social independence domains of the SWPER index had lower DFPS with permanent methods, but higher with SARC methods. Regarding the DFPS with permanent methods, there was a 13.2 percentage point gap between the low vs. highly empowered women in attitude to violence $(84.0 \%$ vs $70.8 \%$, respectively) and a 22.5 percentage point gap in the social independence domain $(87.0 \%$ vs $64.5 \%$, respectively). Even though DFPS was lower among more empowered women in these domains, they presented a higher share of SARC and LARC methods, while less empowered women more frequently relied on permanent methods. The decision-making domain showed

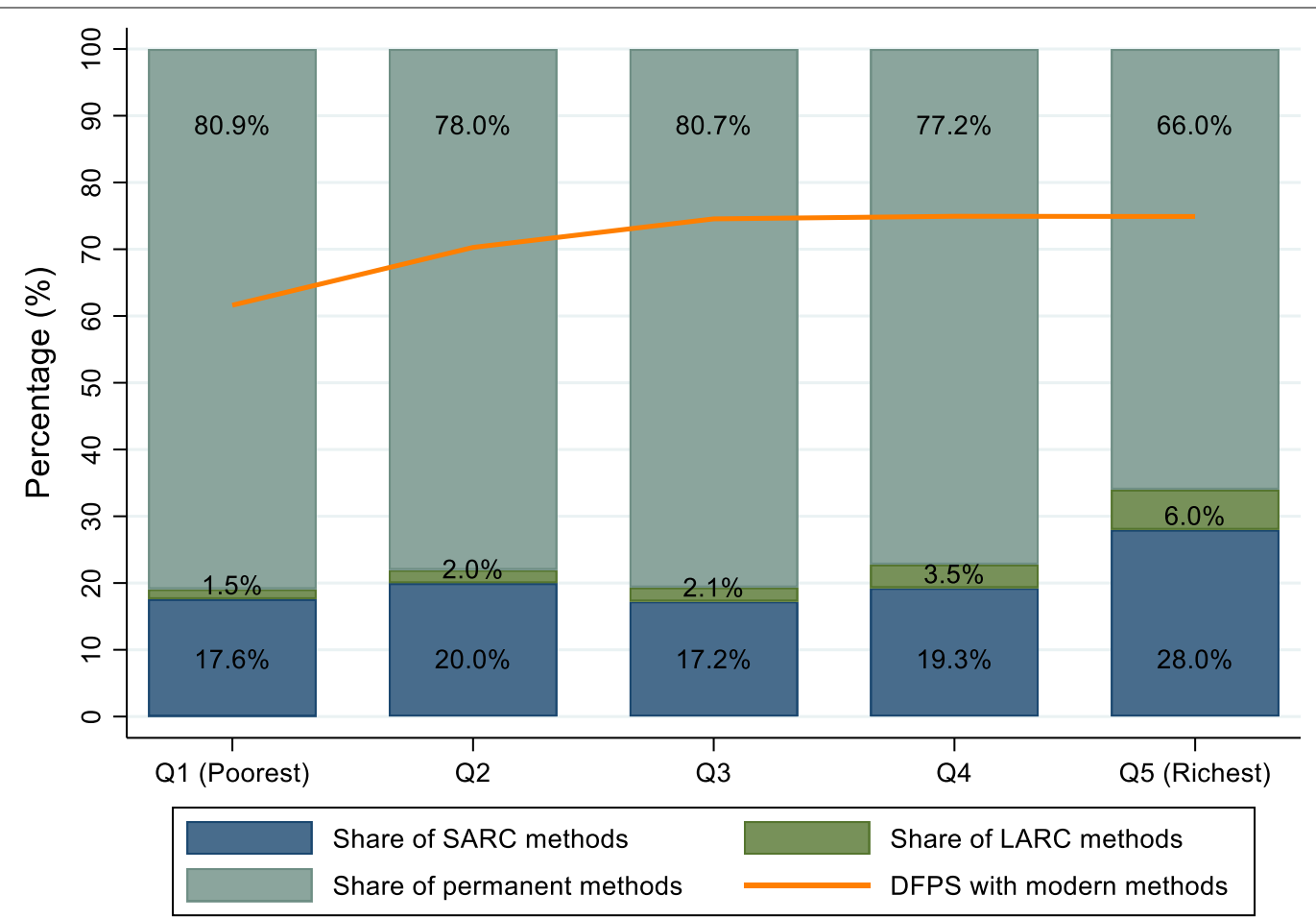

Fig. 2 Demand for family planning satisfied (DFPS) with modern methods and the share of each type of contraceptive being used among partnered women aged 15-49 currently using contraception according to household wealth quintiles. India, 2015-2016 ( N=339,540). Note Short-acting reversible contraceptive (SARC) methods include condoms (male and female), injectables, diaphragms, jelly or foam and emergency contraception; Long-acting reversible contraceptive (LARC) methods include intrauterine devices (IUD); and Permanent methods include male and female sterilization 


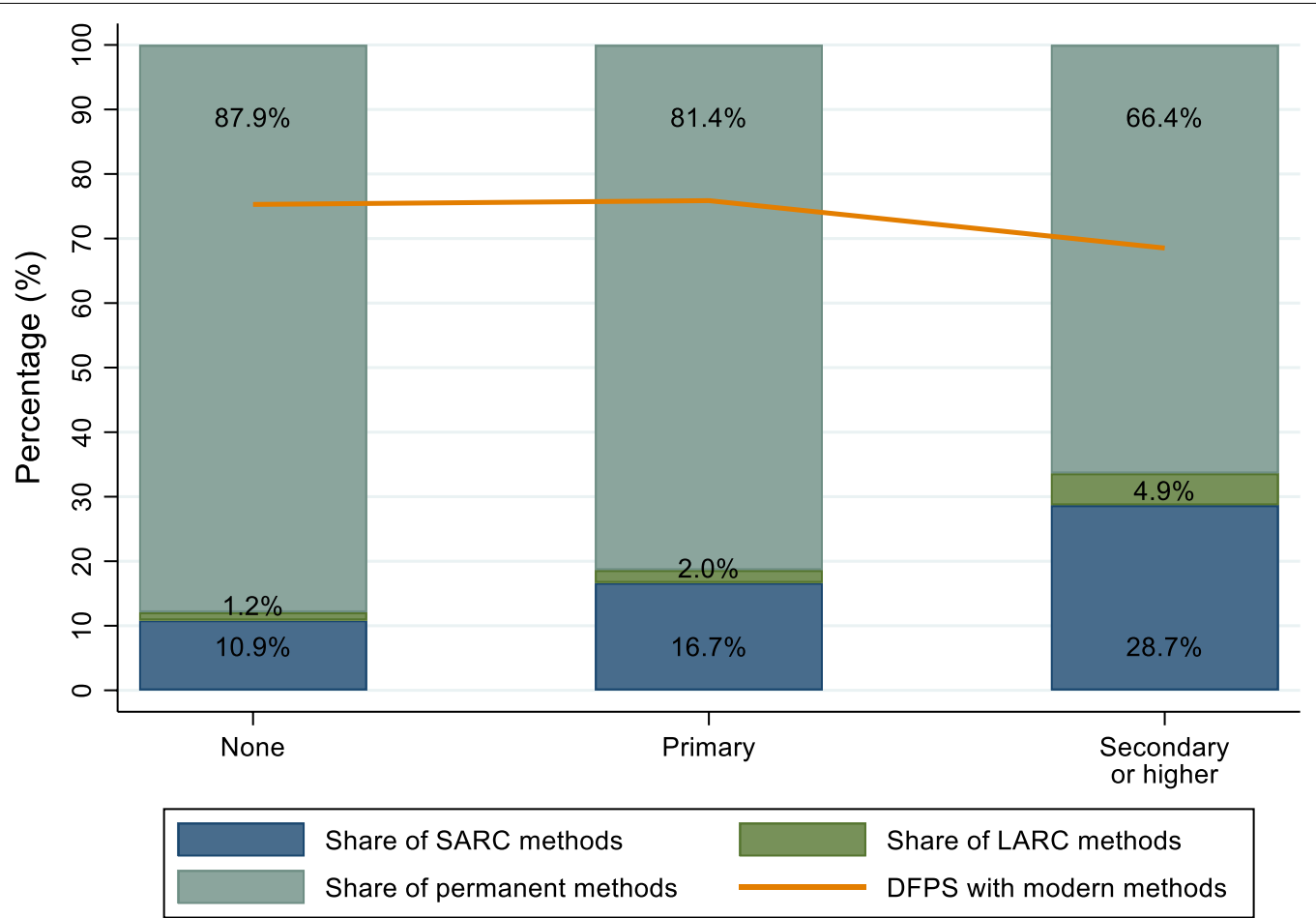

Fig. 3 Demand for family planning satisfied (DFPS) with modern methods and the share of each type of contraceptive being used among partnered women aged 15-49 currently using contraception according to women's education. India, 2015-2016 (N=339,540). Note Short-acting reversible contraceptive (SARC) methods include condoms (male and female), injectables, diaphragms, jelly or foam and emergency contraception; Long-acting reversible contraceptive (LARC) methods include intrauterine devices (IUD); and Permanent methods include male and female sterilization

different patterns, with higher DFPS with permanent methods among the highly empowered women, though the difference was not as marked $(77.2 \%, 95 \%$ CI $75.4-$ 78.9 vs $76.1 \%$, 95\% CI 75.1-77.1) for the low and high empowerment women, respectively). For decision-making, the share of each type of contraceptive used was very similar regardless of the women's empowerment level.

Table 2 shows a description of the women that have undergone sterilization. It shows that $42.9 \%$ (95\% CI 42.4-43.4) of the sterilized women had the procedure before they were aged 25 years old. The vast majority of the women had 2 or more children at time of sterilization; only $3.3 \%, 95 \%$ CI $3.1-3.5$ had none or one child at sterilization. One in five sterilized women $(20.8 \%$, 95\% CI 20.3-21.2) were not told that sterilization would mean no more children, with no difference according to the time since sterilization (results not shown). Even so, the percentage of women who said they regretted sterilization was a modest $6.9 \%$ (95\% CI 6.7-7.2), ranging from 5.8\% (95\% CI 5.5-6.1) among those that had 4 or more children when the procedure was done to $11.8 \%$ (95\% CI 10.3-13.1) among women that had up to one child. Most (61.2\%; 95\% CI 61.0-62.1) of the sterilized women said they received monetary compensation for the sterilization, ranging from $76.3 \%(95 \%$ CI $75.6-77.1)$ among the poorest women to $40.9 \%$ (95\% CI 39.7-42.2) among the richest. The average amount of monetary compensation received was higher among richer women. There was no substantial difference in the proportion of women receiving compensation according to the time since the procedure was done (results not shown).

\section{Discussion}

Findings from this study demonstrate that nearly three in every four $(72 \%)$ partnered women in need of contraception in India are using a modern method. This is a negligible improvement from DFPS observed in 2005 data, which was at 70\% [3]. Further, three-quarters of this use is represented by female sterilization, again demonstrating inadequate progress in the promotion of reversible contraceptive use in the nation in the past decade. Further, $43 \%$ of sterilized women are under 25 years of age, with the fastest increase in sterilization prevalence occurring between ages 19-25 years [8]. This directly corresponds with India's median age at first birth of 21 years, median birth interval of 32 months, and total fertility rate of 2.2 children [8]. India's reliance on female sterilization is largely due 


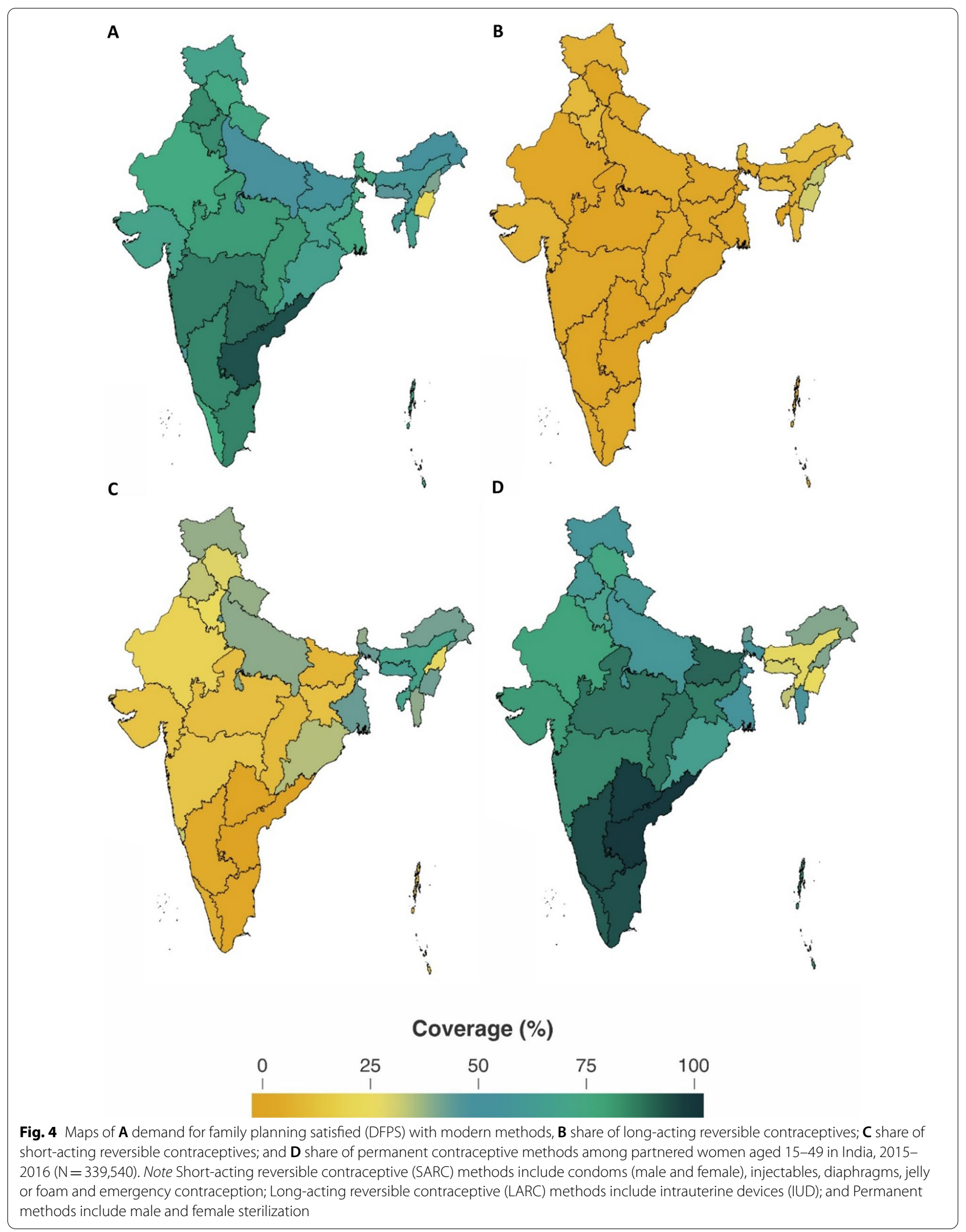


to a historical legacy of government policy that promoted sterilization to control population growth [12, 13], as well as patriarchal norms that view vasectomy as a threat to masculinity and sexuality $[14,15]$. Importantly, there is some indication of increasing modern contraceptive use based on preliminary state level data collected in 2019-2020, under the NFHS-5, although sterilization continues to be the dominant form of contraceptive used [16].

Reliance on SARC or LARC was much less common (20.7\% and $3.2 \%$, respectively). Several factors likely contribute to these low shares. There is an ongoing fear of side effects and health issues associated with the use of different SARC and LARC methods [17]. Additionally, research on contraceptive use in India suggests that familial pressure, as well as gender and social norms, play a strong role and that these norms have shifted little over time [17-19]. Many women do not use contraception following marriage in order to demonstrate their fertility, and as a result of pressure from husbands, in-laws and communities [20-22]. Deviation from these norms may facilitate greater uptake and sustained use of these methods, thus reducing reliance on sterilization. Our results showed that the highest share of SARC and LARC use were found among women with higher socioeconomic status, education and empowerment levels. Even though these subgroups of women presented higher reliance on non-permanent methods, they have lower DFPS with modern methods, which reinforces the difficulty of using contraception that is not sterilization. After infrequent sex, the most common reasons women reported for not using contraception in India were the opposition of the husband or someone else (19.7\%), lack of access (9.8\%), fatalistic approach $(9.1 \%)$, respondent opposition (8.1\%) and health concerns (7.4\%) [19]. Across age groups, LARC use was highly invariant. However, at younger ages, there is a much higher share of SARC methods, that is almost linearly exchanged to permanent methods as women get older. Evidence shows that, even among sexually active unpartnered women, sterilization is the most commonly used contraceptive method in India [8]. We may, however, see a change when new data become available due to the Mission Parivar Vikas, a government initiative launched in 2016 to promote modern contraceptives in 146 high fertility districts via financial incentives for women and family planning providers (financial incentives apply for injectable contraception, IUD and sterilization) [23].

There is evidence to suggest that women from more marginalized backgrounds achieve higher levels of family and community status, as well as greater freedom of movement, only following their sterilization [24]. For these women, therefore, current social structures may impede their ability to access some of the rights enjoyed by wealthier, more educated and more empowered women prior to sterilization. These findings highlight the need not only for more targeted efforts to support access and uptake of SARC and LARC methods among more socially vulnerable women, but also a need to understand in greater detail how normative, structural and economic barriers may affect their contraceptive decision-making. Our results also offer caution regarding sterilization, however. One in five sterilized women were not told that their procedures meant that their childbearing would be complete, and well more than half $(62 \%)$ received financial incentives for undergoing sterilization. In the context of a long history of forced and coercive sterilizations [12], in which sterilization targets and financial incentives for women who undergo sterilization and health workers who enable those procedures [25] still exist, these results indicate a need for a greater understanding of women's information, choices and autonomy regarding these procedures. Sterilization regret, while a concern at any level, was very low in this sample (7\%). However, given the large proportion of women undergoing sterilization, the number of women experiencing regret are considerable [26]. We estimate that more than 92 million women in reproductive age are sterilized in India, thus around 6.5 million women regret having undergone the procedure. Additionally, sterilization levels were similar across wealth quintiles, indicating that incentivization alone is not driving this high prevalence. Given the substantial geographic heterogeneity in the prevalence of demand for family planning satisfied with permanent methods, with higher coverage in the south, central and west of India, and lower coverage in the north and east, in line with previous research, additional geospatial analyses may be warranted [20].

Importantly, this study was able to identify the ways women meet their need for family planning across both types of contraception and domains of empowerment. The least socially independent women (e.g. those with

\footnotetext{
(See figure on next page.)

Fig. 5 Demand for family planning satisfied (DFPS) with modern methods and the share of each type of contraceptive being used among partnered women aged 15-49 currently using contraception according to the women's empowerment level in attitude to violence, social independence, and decision-making domains. India, 2015-2016 ( $\mathrm{N}=59,434)$. Note Short-acting reversible contraceptive (SARC) methods include condoms (male and female), injectables, diaphragms, jelly or foam and emergency contraception; Long-acting reversible contraceptive (LARC) methods include intrauterine devices (IUD); and Permanent methods include male and female sterilization
} 

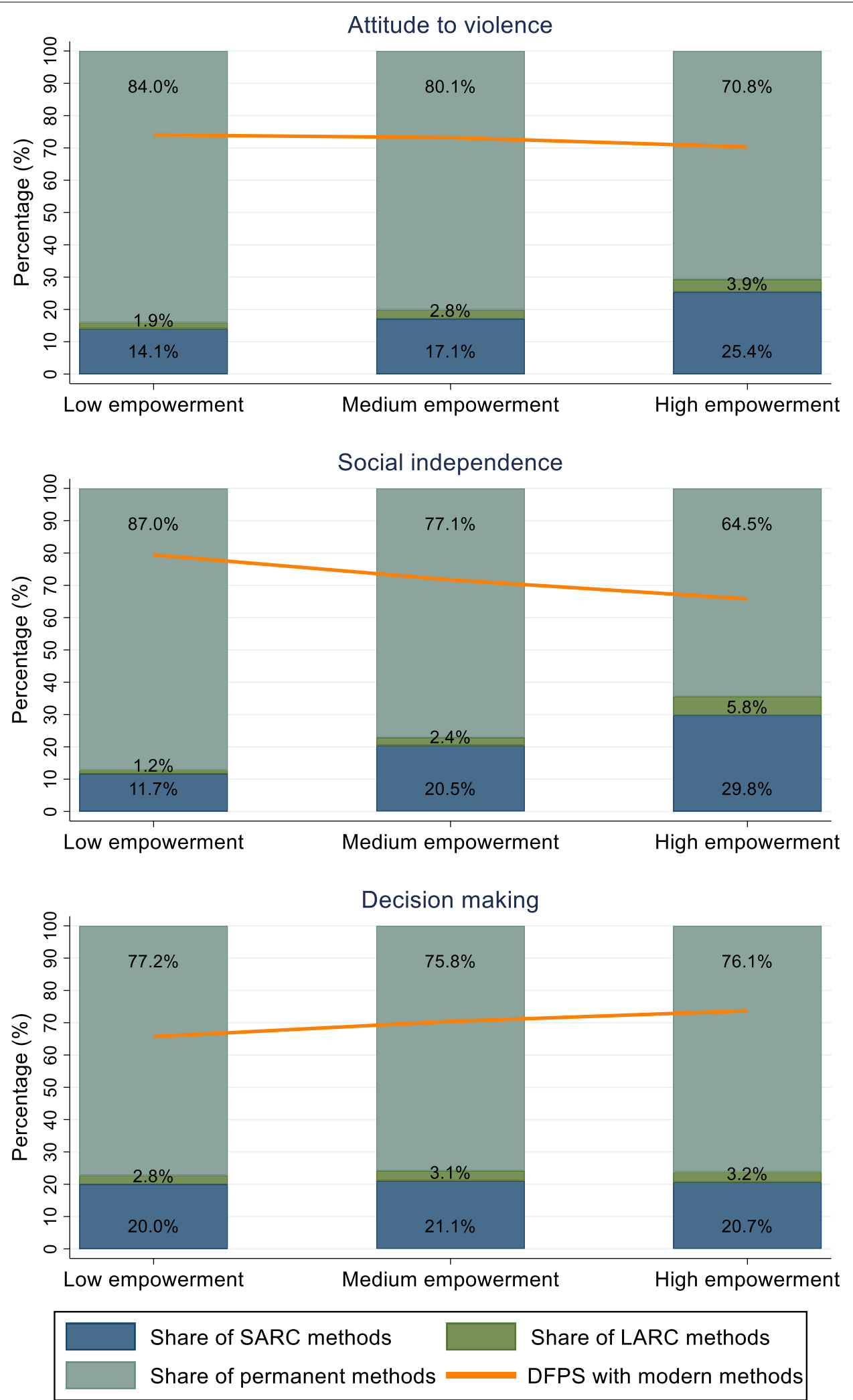

Fig. 5 (See legend on previous page.) 
Table 2 Description of partnered women aged 15-49 years that were sterilized. India, 2015-2016 ( $N=185,429)$

\begin{tabular}{|c|c|c|}
\hline & $\%$ & $95 \% \mathrm{Cl}$ \\
\hline \multicolumn{3}{|l|}{ Age at sterilization } \\
\hline$<25$ years & 42.9 & $42.4-43.4$ \\
\hline $25-29$ years & 35.4 & $35.0-35.8$ \\
\hline 30-34 years & 15.7 & $15.5-16.0$ \\
\hline$>35$ years & 6.0 & $5.8-6.2$ \\
\hline \multicolumn{3}{|l|}{ Parity at sterilization } \\
\hline 0-1 child & 3.3 & $3.1-3.5$ \\
\hline 2 children & 43.4 & $42.8-43.9$ \\
\hline 3 children & 29.4 & $29.0-29.7$ \\
\hline $4+$ children & 24.0 & $23.6-24.4$ \\
\hline Not told sterilization would mean no more children & 20.8 & $20.3-21.2$ \\
\hline Regret sterilization & 6.9 & $6.7-7.2$ \\
\hline \multicolumn{3}{|l|}{ Regret according to parity at sterilization } \\
\hline 0-1 child & 11.8 & $10.3-13.1$ \\
\hline 2 children & 7.5 & $7.2-7.9$ \\
\hline 3 children & 6.5 & $6.1-6.8$ \\
\hline $4+$ children & 5.8 & $5.5-6.1$ \\
\hline Received monetary compensation for sterilization & 61.2 & $61.0-62.1$ \\
\hline \multicolumn{3}{|l|}{ Received monetary compensation by wealth quintiles } \\
\hline Q1 (Poorest) & 76.3 & $75.6-77.1$ \\
\hline Q2 & 71.4 & $70.6-72.1$ \\
\hline Q3 & 66.1 & $65.3-66.9$ \\
\hline Q4 & 57.2 & $56.3-58.1$ \\
\hline Q5 (Richest) & 40.9 & $39.7-42.2$ \\
\hline Average monetary compensation by wealth quintiles & Rupees (.) & $95 \% \mathrm{Cl}$ \\
\hline Q1 (Poorest) & 659.3 & $639.3-679.4$ \\
\hline Q2 & 637.0 & $617.9-656.0$ \\
\hline Q3 & 692.2 & $668.3-716.1$ \\
\hline Q4 & 737.0 & $702.6-771.5$ \\
\hline Q5 (Richest) & 784.0 & $727.7-840.3$ \\
\hline
\end{tabular}

lower levels of information access, educational attainment, and age at first marriage and childbirth, and with higher gaps in age and education between spouses) have the highest reliance on permanent methods. These findings correspond with extensive research from India, consistently showing greater uptake of sterilization and younger age at sterilization among socially marginalized relative to more privileged women [8, 13, 24, 27-29].

This study has some limitations. Data were derived from a self-reported survey and are thus subject to recall bias. Causality cannot be inferred from this observational, cross-sectional analysis. Due to the unique history of contraceptive uptake in India, as well as the current highly skewed method mix, results may have limited generalizability beyond India. Also, we know that contraceptive use has increased since 2015-16, when these data were collected, based on preliminary findings from state level data collected in 2019-2020 [16]. However, the current analyses use the only nationally representative data available from India, and more recent data show similar patterns of contraceptive use as seen in these 2015-16 data, albeit at higher rates.

\section{Conclusions}

This study adds to the growing body of research aimed at explaining the relationship between women's empowerment and contraceptive use. It is clear that empowerment affects the methods with which women satisfy their need for family planning, with women with more gender-equitable views on violence against women and more socially independent being less likely to use permanent methods and more likely to use SARC and LARC methods than their less empowered counterparts. There is a need to make LARC and SARC 
methods more widely available and promoted, which will likely require policy and health system shifts away from target-based incentives and towards women-centered care. Concurrently, there is a need to shift the longstanding norms that sustain the cycle of early marriage, early in marriage fertility, and then sterilization once desired number and sex of children is achieved, something that gender-equity-focused family planning programs involving both men and women show promise in addressing [30-32]. This multilevel approach, involving government, policy makers, and society will be key in supporting the shift towards woman-centered contraceptive service provision that meets the needs of all Indian women.

\section{Abbreviations}

DFPS: Demand for family planning satisfied; IUD: Intrauterine device; LARC : Long-acting reversible contraceptive; NFHS: National Family and Health Survey; PSU: Primary sampling unit; SARC: Short-acting reversible contraceptive; SWPER: Survey-based Women's emPoWerment Index.

\section{Supplementary Information}

The online version contains supplementary material available at https://doi. org/10.1186/s12978-021-01220-w.

Additional file 1. Demand for family planning satisfied (DFPS) and share of each type of contraceptive being used across Indian states and union territories, 2015-16.

\section{Acknowledgements}

This paper builds on analysis included in the WHO Universal Health Coverage Global Monitoring Report 2019. This report was reviewed by many experts including from the WHO Regional Office in India. The findings were also presented at a Seminar in WHO Geneva on 2 Dec 2019 and at a side event of the United Nations General Assembly 74 UHC High Level Meeting on 24th Sept 2019, which had panel members and attendees from India. The authors would also like to thank the reviewers of this report, participants of the seminars, as well as colleagues from the International Institute for Population Sciences (IIPS) for their insights into NFHS-4 data and contraceptive use preferences and dynamics in India. We would also like to thank Veronica Magar (Gender, Equity and Human Rights Team, World Health Organization (WHO), Geneva, Switzerland) for her valuable contributions to this study and Cintia Borges (International Center for Equity in Health) for assisting with the graphs design.

\section{Authors' contributions}

FE, LM, CB and LF conducted the analysis and drafted the manuscript. AR and DP contributed to the writing of the manuscript and the critical revision of the analysis. AB advised on the analysis and contributed to the writing and revision of the manuscript. All authors read and approved the final manuscript.

\section{Funding}

The study was funded by the World Health Organization, Bill \& Melinda Gates Foundation [Grant Number: OPP1199234] and Wellcome [Grant Number: $101815 / Z / 13 / Z]$. The funders of the study had no role in the data analysis, data interpretation, or writing of the paper. The corresponding author had full access to all the data and had final responsibility for the decision to submit it for publication.

\section{Availability of data and materials}

NFHS-4 data is publicly available at https://dhsprogram.com/data/availabledatasets.cfm

\section{Declarations}

\section{Ethics approval and consent to participate}

This study used data from NFHS-4, which is a publicly available source of data, so ethical clearance was not required for this study. The NFHS-4 study protocol, including all the survey questionnaires, was approved by the International Institute for Population Sciences Institutional Review Board and the ICF Institutional Review Board.

\section{Consent for publication}

Not applicable.

\section{Competing interests}

The authors declare no competing interests.

\section{Author details}

${ }^{1}$ International Center for Equity in Health, Universidade Federal de Pelotas, Pelotas, Brazil. ${ }^{2}$ Center on Gender Equity and Health, University of California San Diego, San Diego, USA. ${ }^{3}$ Postgraduate Program in Epidemiology, Universidade Federal de Pelotas, Pelotas, Brazil. ${ }^{4}$ King's Centre for Global Health and Health Partnerships, School of Population Health and Environmental Sciences, King's College London, London, UK. ${ }^{5}$ Marechal Deodoro 1160, 3rd floor, Pelotas, RS, Brazil.

Received: 14 January 2021 Accepted: 5 August 2021

Published online: 21 August 2021

\section{References}

1. Blumenberg C, Hellwig F, Ewerling F, Barros AJD. Socio-demographic and economic inequalities in modern contraception in 11 low- and middleincome countries: an analysis of the PMA2020 surveys. Reprod Health. 2020;17(1):82. https://doi.org/10.1186/s12978-020-00931-w.

2. Ponce de Leon RG, Ewerling F, Serruya SJ, Silveira MF, Sanhueza A, Moazzam A, et al. Contraceptive use in Latin America and the Caribbean with a focus on long-acting reversible contraceptives: prevalence and inequalities in 23 countries. Lancet Glob Health. 2019;7(2):e227-35.

3. Ewerling F, Victora CG, Raj A, Coll CVN, Hellwig F, Barros AJD. Demand for family planning satisfied with modern methods among sexually active women in low- and middle-income countries: who is lagging behind? Reprod Health. 2018;15(1):1-10.

4. Maru RM. Incentives and disincentives in the Indian family welfare program. Stud Fam Plann. 1986;17(3):136-45.

5. Chatterjee N, Riley NE. Planning an Indian modernity: the gendered politics of fertility control. Signs J Women Cult Soc. 2001;26(3):811-45.

6. Government of India. India's Vision FP 2020 [Internet]. New Delhi; 2014. http://www.familyplanning2020.org/sites/default/files/Indias-VisionFP2020.pdf

7. World Health Organization. Primary Health Care on the Road to Universal Health Coverage: 2019 Global Monitoring Report. Geneva WHO. 2019

8. International Institute for Population Sciences (IIPS). National Family Health Survey (NFHS-4), 2015-16. IIPS. Mumbai, India; 2017.

9. Bradley SEK, Croft TN, Fishel JD, Westoff CF. Revising Unmet Need for Family Planning: DHS Analytical Studies No. 25. Rockville, MA: ICF International; 2012.

10. Hubacher D, Trussell J. A definition of modern contraceptive methods. Contraception. 2015;92(5):420-1. https://doi.org/10.1016/j.contraception. 2015.08.008.

11. Ewerling F, Raj A, Victora CG, Hellwig F, Coll CVN, Barros AJD. SWPER Global: a survey-based women's empowerment index expanded from Africa to all low- and middle-income countries. medRxiv. 2020;10:2.

12. Gupte PR. India: "The Emergency" and the politics of mass sterilization. Educ About Asia. 2017;22(3):40-4.

13. De Oliveira IT, Dias JG, Padmadas SS. Dominance of sterilization and alternative choices of contraception in India: an appraisal of the socioeconomic impact. PLoS ONE. 2014;9(1):e86654.

14. Mahapatra S, Narula C, Thakur CP, Kalita TJ, Mehra R. Assessment of knowledge and perception regarding male sterilization (non-scalpel 
vasectomy) among community health workers in Jharkhand, India. Indian J Commun Health. 2014;26(4):428-33.

15. Hall MAK, Stephenson RB, Juvekar S. Social and logistical barriers to the use of reversible contraception among women in a rural Indian village. J Health Popul Nutr. 2008;26(2):241

16. International Institute for Population Sciences (IIPS). National Family Health Survey (NFHS-5): key indicators 22 states/UTs from phase-I. New Delhi; 2020. http://www.rchiips.org/NFHS/NFHS-5_FCTS/NFHS-5Stat eFactsheetCompendium_Phase-I.pdf

17. Ghule M, Raj A, Palaye P, Dasgupta A, Nair S, Saggurti N, et al. Barriers to use contraceptive methods among rural young married couples in Maharashtra, India: qualitative findings. Asian J Res Soc Sci Humanit. 2015:5(6):18.

18. Khanna T, Chandra M, Singh A, Mehra S. Why ethnicity and gender matters for fertility intention among married young people: a baseline evaluation from a gender transformative intervention in rural India. Reprod Health. 2018;15(1):63.

19. Moreira LR, Ewerling F, Barros AJD, Silveira MF. Reasons for nonuse of contraceptive methods by women with demand for contraception not satisfied: an assessment of low and middle-income countries using demographic and health surveys. Reprod Health. 2019;16(1):148.

20. Jejeebhoy SJ, Santhya KG, Zavier AJF. Demand for contraception to delay first pregnancy among young married women in India. Stud Fam Plann. 2014:45(2):183-201.

21. Barua A, Kurz K. Reproductive health-seeking by married adolescent girls in Maharashtra, India. Reprod Health Matters. 2001;9(17):53-62.

22. McClendon KA, McDougal L, Ayyaluru S, Belayneh Y, Sinha A, Silverman $J G$, et al. Intersections of girl child marriage and family planning beliefs and use: qualitative findings from Ethiopia and India. Cult Health Sex. 2018;20(7):799-814.

23. Ministry of Health \& Family Welfare - Government of India. Mission Parivar Vikas guidelines. p. 23. http://nhm.gov.in/images/pdf/programmes/family-planing/guidelines/MPV/MPV_guidelines.pdf
24. Saavala M. Understanding the prevalence of female sterilization in rural South India. Stud Fam Plann. 1999;30(4):288-301.

25. Sharma DC. India's sterilisation scandal. Lancet. 2014;384(9961):e68-9.

26. Singh A, Ogollah R, Ram F, Pallikadavath S. Sterilization regret among married women in India: implications for the Indian national family planning program. Int Perspect Sex Reprod Health. 2012;15:187-95.

27. Khan ME, Prasad CVS. Fertility control in India: a critical evaluation of the role of incentives in promoting sterilization among industrial workers. Manohar; 1980.

28. Desai S, Campbell OMR, Sinha T, Mahal A, Cousens S. Incidence and determinants of hysterectomy in a low-income setting in Gujarat, India. Health Policy Plan. 2017;32(1):68-78.

29. Raj A, Saggurti N, Balaiah D, Silverman JG. Prevalence of child marriage and its effect on fertility and fertility-control outcomes of young women in India: a cross-sectional, observational study. Lancet. 2009:373(9678):1883-9.

30. Shakya HB, Dasgupta A, Ghule M, Battala M, Saggurti N, Donta B, et al. Spousal discordance on reports of contraceptive communication, contraceptive use, and ideal family size in rural India: a cross-sectional study. BMC Womens Health. 2018;18(1):147.

31. Dixit A, Averbach S, Yore J, Kully G, Ghule M, Battala M, et al. A gender synchronized family planning intervention for married couples in rural India: study protocol for the CHARM2 cluster randomized controlled trial evaluation. Reprod Health. 2019;16(1):88.

32. Raj A, Ghule M, Ritter J, Battala M, Gajanan V, Nair S, et al. Cluster randomized controlled trial evaluation of a gender equity and family planning intervention for married men and couples in rural India. PLoS ONE. 2016;11(5):e0153190.

\section{Publisher's Note}

Springer Nature remains neutral with regard to jurisdictional claims in published maps and institutional affiliations.
Ready to submit your research? Choose BMC and benefit from:

- fast, convenient online submission

- thorough peer review by experienced researchers in your field

- rapid publication on acceptance

- support for research data, including large and complex data types

- gold Open Access which fosters wider collaboration and increased citations

- maximum visibility for your research: over $100 \mathrm{M}$ website views per year

At BMC, research is always in progress.

Learn more biomedcentral.com/submissions 